\title{
Transformación de una línea de montaje de modelos mixtos en un taller de flujo regu- lar: Caso de estudio en la factoría Nissan de Barcelona
}

\author{
Joaquín Bautista, Rocío Alfaro \\ Recibido: 13 de Septiembre de 2019 / Aceptado: 1 de Noviembre de 2019
}

\section{Resumen}

En este trabajo aplicamos dos modelos de Programación lineal entera mixta, sobre problemas de taller de flujo regular con demanda generalizada de tipos de trabajos, con el propósito de transformar una línea de modelos mixtos en un taller de flujo regular. Para ilustrar dicha aplicación, presentamos un caso de estudio basado en una línea de motores mixtos de la factoría de Nissan en Barcelona. En el estudio se comparan los costes de producción de los dos sistemas productivos enfrentados, llegando a la conclusión de que el sistema taller de flujo regular sin bloqueos entre estaciones es el más competitivo. Como herramienta de resolución hemos empleado el solucionador CPLEX con resultados satisfactorios. Para llevar a la práctica la transformación de la línea de producción en un taller de flujo regular, proponemos un conjunto de requisitos, así como la asistencia al sistema productivo con un sistema de información basado en tecnologías IoT en el marco de la Industria 4.0.

\section{Palabras clave}

Taller de flujo regular, Secuenciación de modelos mixtos, Líneas de ensamblado de modelos mixtos, Plan de demanda general, Programación lineal entera mixta, Industria 4.0.

\section{Introducción}

El problema de secuenciación de trabajos (piezas, tareas, operaciones, etc.) en un taller de flujo regular (FSP: Flow Shop Scheduling Problem) permite modelar una gran variedad de contextos productivos (Pinedo 2016), por lo que se ha convertido en las últimas décadas en una de las familias de problemas de secuenciación que ha recibido más atención en entornos profesionales y de investigación.

Formalmente, en el FSP, un conjunto J de trabajos o productos (n elementos) son procesados en un conjunto de máquinas $\mathrm{K}$ (m elementos) dispuestas en serie que constituyen el taller de fabricación. Todos los trabajos deben pasar por todas las máquinas en el mismo orden, empezando en la máquina 1 y finalizando en la máquina $\mathrm{m}$.

El trabajo $j \in J(j=1, . ., n)$ requiere un tiempo de proceso $\mathrm{p}_{\mathrm{j}, \mathrm{k}} \geq 0$ en la máquina $\mathrm{k} \in \mathrm{K}(\mathrm{k}=1, . ., \mathrm{m})$. El objetivo general del FSP es establecer una secuencia de lanzamientos de los trabajos en el taller que optimice uno o varios criterios de eficiencia.

\begin{tabular}{|c|c|}
\hline \multirow[t]{6}{*}{ 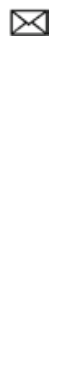 } & $\begin{array}{l}\text { Joaquín Bautista * } \\
\text { joaquin.bautista@upc.edu }\end{array}$ \\
\hline & ORCID: 0000-0002-2214-4991 \\
\hline & $\begin{array}{l}\text { Rocío Alfaro ** } \\
\text { ralfaro@eae.es }\end{array}$ \\
\hline & ORCID: 0000-0001-8214-1875 \\
\hline & $\begin{array}{l}\text { * IOC - División de Robótica, Universitat Politècnica de } \\
\text { Catalunva, } 08028 \text { Barcelona, Spain }\end{array}$ \\
\hline & ** EAE Business School, 08015 Barcelona, Spain \\
\hline
\end{tabular}

La versión del problema denominada Permutation Flow Shop Problem (PFSP), considera que el espacio de almacenamiento entre dos máquinas consecutivas es ilimitado, por lo que se da por supuesto que, una vez concluida la operación $(\mathrm{j}, \mathrm{k})$ sobre el trabajo $\mathrm{j} \in \mathrm{J}$ en la máquina $\mathrm{k}>1(\mathrm{k} \in \mathrm{K})$, ésta quedará libre para poder procesar el siguiente trabajo de la secuencia a partir del instante en que dicho trabajo sea liberado por la máquina anterior k-1. Haciendo uso de la notación propuesta por Graham et al. (1979), este problema se conoce como Fm/prmu/ $\gamma$, donde el parámetro $\gamma$ simboliza la medida de eficiencia seleccionada. Entre las métricas de eficiencia más comunes están: (1) el makespan o instante de finalización de todas las operaciones $(\mathrm{j}, \mathrm{k})$ en el taller, simbolizado por $\mathrm{C}_{\max }$, $\mathrm{y}(2)$ el instante medio de finalización de dichas operaciones, simbolizado por $\mathrm{C}_{\text {med }}$.

Es evidente que hay sistemas productivos, formados también por máquinas o células de fabricación dispuestas en serie, en los que no es posible apartar los productos o los trabajos del proceso, ya sea por la envergadura de dichos productos (v.gr.: bastidores, autobuses), por la naturaleza de los trabajos (v.gr.: reactores químicos), o, simplemente, porque no se puede debido a la falta de espacio. Ante tal circunstancia, una vez concluida la operación sobre un producto en la máquina $\mathrm{k}<\mathrm{m}$, ésta deberá liberar dicho producto hacia la máquina siguiente $\mathrm{k}+1$ para poder procesar el siguiente producto de la secuencia, pero si la máquina k+1 está ocupada, es obvio que la máquina k quedará bloqueada a pesar de que esta haya concluido su operación. Haciendo uso otra vez de la notación de Graham et al. (1979), este problema se denomina $\mathrm{Fm} / \mathrm{block} / \gamma$, donde $\gamma$ simboliza de nuevo la medida de eficiencia. 
La naturaleza de estos problema es altamente combinatoria y la minimización del makespan es NP-hard en sentido fuerte (Hoogeveen et al. 1996; Hall and Sriskandarajah 1996; Yu et al. 2004), por ello, tradicionalmente, se han utilizado las heurísticas para resolverlos, tanto es su versión permutación (Nawaz et al. 1983; Osman and Potts 1989; Taillard 1990, 1993; Reeves 1995; Aggoune 2004; Ying and Liao 2004; Fernandez-Viagas et al. 2017) como en su versión con bloqueo entre máquinas (Logendran and Sriskandarajah 1993; Caraffa et al. 2001; Ronconi 2004, 2005; Ribas et al. 2011; Grabowski and Pempera 2007; Han et al. 2012; Pan and Wang 2012; Lin and Ying 2013; Nouri and Ladhari 2018, Ozolins 2019; Tasgetiren et al. 2017).

Una vez expuestos los dos problemas que son objeto de nuestro interés, haremos algunas consideraciones sobre el carácter poco realista que, a nuestro parecer, afecta al conjunto J de trabajos en algunos sectores industriales. Concretamente, aquí nos centraremos en el sector de automoción, donde la presencia masiva de las líneas de montaje y los talleres de fabricación, así como la estandarización de los productos forman parte de la cultura del sector.

\section{En efecto:}

- Tradicionalmente, en las diversas versiones del FSP, los elementos del conjunto de trabajos J son singulares, sin cualidades comunes, es decir: son trabajos o productos únicos.

- $\quad$ Si atendemos a algunos sectores industriales, pongamos por caso el de automoción, es difícil encontrar problemas realistas cuyo propósito sea establecer secuencias eficientes de 270 o más productos distintos. De hecho, es absurdo pensar en una secuencia diaria de 270 motores o 300 carrocerías o 500 bastidores o 2100 automóviles (como es el caso de SEAT Martorell) que sean completamente distintos.

- Por lo anterior y a efectos prácticos, es razonable pensar que se puede establecer de forma natural una tipología sobre el conjunto J de trabajos (productos, piezas, etc.), y se podrá hablar, por tanto, de tipos de motores o carrocerías o bastidores o coches.

- En definitiva, en algunos sectores industriales, tiene sentido hablar de tipos de trabajos o de tipos de productos o de tipos de piezas.

El presente estudio es una extensión de un trabajo anterior (Bautista-Valhondo and Alfaro-Pozo 2018a) enfatizando en aspectos económico-tecnológicos. Nuestra propuesta concreta consiste en comparar desde una óptica de costes de producción dos tipos de sistemas productivos:
1 Líneas de montaje de modelos mixtos, donde los distintos productos circulan, uno tras otro, a través del sistema a cadencia constante. Dicho ritmo está marcado por el tiempo de ciclo c que es simplemente el tiempo concedido a cada estación para completar cada trabajo.

2 Talleres de flujo regular (flow shop) constituidos por máquinas simples o por células de fabricación más complejas, las cuales también pueden estar dispuestas en serie emulando a las líneas de ensamblado, pero con la particularidad de que cada puesto de trabajo actúa de forma individual sin estar sujeto a los condicionantes impuestos por un ritmo común.

En el segundo caso existen las opciones de permitir el almacenamiento intermedio de unidades entre pares de células consecutivas, dando así la posibilidad de apartar los productos del flujo continuo de fabricación, o, bien, eliminar dichos espacios intermedios propiciando el bloqueo entre células o estaciones de trabajo consecutivas.

Las principales aportaciones de este estudio son:

1 Formulación de modelos de programación lineal entera mixta (MILP: Mixed Integer Linear Programming) para la secuenciación en talleres de flujo regular con demanda generalizada y su explotación para un caso de estudio en la planta de motores de Nissan en Barcelona.

2 Evaluación de costes de producción en líneas de montaje de modelos mixtos y talleres de flujo regular, abordando el análisis comparativo de ahorros y sobrecostes entre los dos tipos de sistemas productivos: línea de montaje y taller de flujo.

3 Hipótesis y descripción de requerimientos tecnológicos para propiciar la transformación de una línea de montaje de modelos mixtos en un taller de flujo regular.

Tras estas consideraciones, el resto de este documento se estructura como sigue. En la Sección 2, formalizamos una extensión natural del problema del taller de flujo regular cuando existen réplicas de los trabajos, lo cual equivale a decir que existen planes de demanda para los tipos de trabajos. En la Sección 3, recogemos dos modelos de programación lineal entera mixta que modelan estos problemas. La Sección 4 se dedica a un caso de estudio en la factoría de motores de Nissan en Barcelona, donde comparamos los costes en dos sistemas productivos: línea de modelos mixtos y taller de flujo regular, y describimos las condiciones para transformar el primer sistema en el segundo con el uso de tecnologías IoT en el marco de la Industria 4.0. Finalmente, la Sección 5 está dedicada a las conclusiones e investigaciones futuras. 


\section{Definición del problema $\mathbf{F m} / \boldsymbol{\beta} / \gamma / \mathbf{d}_{\mathrm{i}}$ : $\mathrm{Fm} / \boldsymbol{\beta} / \gamma$ con tipos de productos}

Definimos Fm $/ \beta / \gamma /$ di como la familia de problemas de secuencias consistente en establecer una aplicación biyectiva entre los elementos de un conjunto $\mathrm{T}$ de ordinales ( $\mathrm{T}$ elementos), que corresponden a posiciones en una secuencia de lanzamiento a fabricación: $\pi(\mathrm{T})=\left(\pi_{1}, ., \pi_{\mathrm{T}}\right)$, y los elementos de un conjunto $\mathrm{J}$ de trabajos o productos (D elementos, con $\mathrm{D}=\mathrm{T})$.

Los trabajos o productos del conjunto $\mathrm{J}$ se agrupan en tipos o clases excluyentes, $J_{i}$, que cumplen: $J=U_{i \in I} J_{i}$ y $J_{i} \cap$ $\mathrm{J}_{\mathrm{i}}^{\prime}=\emptyset, \forall\{\mathrm{i}, \mathrm{i}\} \in \mathrm{I}$; siendo I el conjunto de tipos de trabajos $(\mathrm{i}=1, . ., \mathrm{n})$. El parámetro $\beta$ puede adoptar los valores permutación (prmu) o bloqueo (block), el parámetro $\gamma$ representa las posibles métricas de eficiencia (v.gr. $\mathrm{C}_{\max }, \mathrm{C}_{\text {med }}$ ), el vector $\vec{d}$ representa el plan de demanda de tipos de trabajo, $\mathrm{y} \mathrm{d}_{\mathrm{i}}$ simboliza el número de trabajos de tipo $\mathrm{i} \in \mathrm{I}$ contenidos en J; obviamente: $\mathrm{d}_{\mathrm{i}}=\left|\mathrm{J}_{\mathrm{i}}\right| \forall \mathrm{i} \in \mathrm{I}$, cumpliéndose: $\sum_{\forall \mathrm{i}} \mathrm{d}_{\mathrm{i}}=\mathrm{D}=\mathrm{T}$.

Las unidades de $\mathrm{J}$ circulan en fila a través de un conjunto $\mathrm{K}$ de $\mathrm{m}$ máquinas dispuestas en serie. Supondremos que el trabajo de tipo i $\in$ I requiere para su elaboración un tiempo de proceso $\mathrm{p}_{\mathrm{i}, \mathrm{k}}$, que se mide a actividad normal, en la máquina $\mathrm{k} \in \mathrm{K}(\mathrm{k}=1, . ., \mathrm{m})$, y que dichos tiempos son heterogéneos.

En efecto, las diferencias entre las clases $J_{\mathrm{i}}$ (v.gr. SUV, Van, Truck) marcan la heterogeneidad de los tiempos de proceso $\mathrm{p}_{\mathrm{i}, \mathrm{k}}$, lo cual se traduce en desacoplamientos naturales entre los procesadores (operarios y robots) asignados a las máquinas. En algunas ocasiones, los operarios deberán esperar a que un producto en curso sea liberado desde la máquina anterior para trabajar sobre él, y en otras, cuando no sea posible el almacenamiento entre máquinas, el operario, aun habiendo terminado su operación sobre el producto en curso, tendrá que esperar "bloqueado” a que se complete la operación que está en curso en la máquina siguiente. Con la descripción anterior, dejamos claro que en este documento no vamos a contemplar la posibilidad de interrumpir las operaciones, reservando esta opción para futuros trabajos.

En cualquier caso, el propósito de los problemas $\mathrm{Fm} / \mathrm{\beta} / \gamma /$ $\mathrm{d}_{\mathrm{i}}$ es obtener una secuencia de trabajos o productos replicados $\left(\mathrm{d}_{\mathrm{i}}\right)$, en una línea con m máquinas, posibilidad de bloqueo o no según $\beta$, y con el objetivo de optimizar la métrica de eficiencia representada por el valor que adopte $\gamma$. Para formalizar dicho propósito, aquí presentamos dos modelos matemáticos adaptados a la programación lineal entera mixta (MILP).

\section{Modelos MILP para los problemas $\mathbf{F m} / \boldsymbol{\beta} / \gamma / \mathbf{d}_{\mathrm{i}}$}

\subsection{Nomenclatura básica}

Parámetros:

J Conjunto de trabajos o productos (Jobs): $\mathrm{j}=1$,.,D

T Conjunto de posiciones en la secuencia de fabricación de los productos: $\mathrm{t}=1$, ,., $\mathrm{T}$

I Conjunto de tipos de trabajos o productos (Job Typos): $\mathrm{i}=1, ., \mathrm{n}$

$\mathrm{J}_{\mathrm{i}} \quad$ Conjunto de trabajos o productos de tipo $\mathrm{i} \in \mathrm{I}$

$\overrightarrow{\mathrm{d}} ; \mathrm{d}_{\mathrm{i}}$ Vector plan de demanda de tipos de trabajos; y demanda de los trabajos o productos de tipo i $(\mathrm{i}=1, ., \mathrm{n})$, con $\mathrm{d}_{\mathrm{i}}=\left|\mathrm{J}_{\mathrm{i}}\right| \forall \mathrm{i} \in \mathrm{I}$ y cumpliéndose: $\sum_{\forall \mathrm{i}} \mathrm{d}_{\mathrm{i}}=\mathrm{D}=\mathrm{T}$

$\mathrm{K}$ Conjunto de máquinas: $\mathrm{k}=1$,.,m. En este trabajo usaremos indistintamente los términos máquina, célula y estación de trabajo para referirnos a este conjunto.

$\mathrm{p}_{\mathrm{i}, \mathrm{k}}$ Tiempo de proceso de un trabajo o producto de tipo $\mathrm{i} \in \mathrm{I}$ en la máquina $\mathrm{k} \in \mathrm{K}$

$\mathrm{C}^{0}{ }_{\text {max }}$ Cota superior del makespan o mínimo instante de compleción de los trabajos. Se puede calcular con una secuencia de referencia $\pi^{0}(\mathrm{~T})$ obtenida heurísticamente.

Variables:

$\mathrm{x}_{\mathrm{i}, \mathrm{t}} \quad$ Variable binaria que vale 1 si un trabajo o producto de tipo i $\in$ I se lanza a fabricación en la t-ésima posición $(\mathrm{t}=1, ., \mathrm{T})$, y vale 0 en caso contrario

$\pi(\bullet)$ Secuencia parcial, $\pi(t)=\left(\pi_{1}, \cdot, \pi_{t}\right)$, y secuencia completa, $\pi(\mathrm{T})=\left(\pi_{1}, ., \pi_{\mathrm{T}}\right)$, de fabricación de los trabajos o productos $j \in \mathrm{J}$

$\rho_{k, t} \quad$ Tiempo de proceso del t-ésimo trabajo $\pi_{t}$ de la secuencia de fabricación $\pi(\mathrm{T})$ en la máquina $\mathrm{k} \in \mathrm{K}$

$\mathrm{C}_{k, t}$ Instante de compleción del t-ésimo trabajo $\pi_{t}$ de la secuencia de fabricación $\pi(\mathrm{T})$ en la máquina $\mathrm{k} \in \mathrm{K}$. Si se contempla la posibilidad de bloqueos, $\mathrm{C}_{\mathrm{k}, \mathrm{t}}$ simboliza el instante de liberación del trabajo o producto $\pi_{t} \in \pi(\mathrm{T})$ en la máquina $\mathrm{k} \in \mathrm{K}$

$\mathrm{C}_{\max }$ Makespan: Instante de compleción del último trabajo o producto $\pi_{\mathrm{T}}$ de la secuencia de fabricación $\pi(\mathrm{T})$, en la última máquina $(\mathrm{k}=\mathrm{m})$; esto es: $\mathrm{C}_{\max }=\mathrm{C}_{\mathrm{m}, \mathrm{T}}$ 


\subsection{Modelo MILP para el problema Fm/prmu/ $\mathrm{C}_{\max } / \mathbf{d}_{\mathrm{i}}$}

El primer modelo matemático corresponde al caso en que existen almacenes intermedios (buffers) entre parejas de máquinas consecutivas, teniendo como objetivo optimizar la métrica de eficiencia representada por $\mathrm{C}_{\max }$. Este es:

MILP-1 Fm $/ \mathrm{prmu} / C_{\max } / d_{i}: \quad \min C_{\max } \equiv \min C_{m, T}$

Sujeto a:

$\sum_{i=1}^{n} x_{i, t}=1$

$\forall t=1, ., T$

$\sum_{t=1}^{T} x_{i, t}=d_{i}$

$\forall i=1, ., n$

$\rho_{k, t}=\sum_{i=1}^{n} p_{i, k} x_{i, t}$

$\forall k=1, ., m$

$\forall t=1, ., T$

$C_{k, t} \geq C_{k, t-1}+\rho_{k, t}$

$\forall k=1, ., m$

$\forall t=1, . ., T$

$C_{k, t} \geq C_{k-1, t}+\rho_{k, t}$

$\forall k=1, . ., m$

$\forall t=1, . ., T$

$X_{i, t} \in\{0,1\}$

$\forall i=1, ., n$

$\forall t=1, . ., T$

$C_{k, 0}=0$

$\forall k=1, ., m$

$C_{0, t}=0$

$\forall \mathrm{t}=1, ., T$

En el modelo MILP-1, la función objetivo (1) representa la minimización del makespan; las igualdades (2) sirven para garantizar una posición en la secuencia a todo trabajo o producto; las igualdades (3) se emplean para imponer la satisfacción del plan de demanda (d); las igualdades (4) vinculan el tiempo de proceso de cada tipo de producto y máquina $\left(\mathrm{p}_{\mathrm{i} k}\right)$ con el correspondiente tiempo de proceso del t-ésimo trabajo de la secuencia $\left(\rho_{\mathrm{k}, \mathrm{t}}\right)$; las restricciones (5) y (6) sirven para acotar inferiormente los instantes de compleción de los trabajos $\left(C_{k, t}\right)$, según la secuencia de fabricación $\pi(T)$, en las máquinas del conjunto $\mathrm{K}$; las condiciones (7) imponen que las variables de decisión $\left(\mathrm{x}_{\mathrm{it}, \mathrm{s}}\right)$ sean binarias; y finalmente (8) y (9) establecen la inicialización de los instantes de compleción.

\subsection{Modelo MILP para el problema Fm/block/ $\mathbf{C}_{\max } / \mathbf{d}_{\mathrm{i}}$}

El segundo modelo representa un taller de flujo regular sin almacenamiento intermedio, por lo que contempla la posibilidad de bloqueo entre estaciones de trabajo consecutivas. Este es:

MILP-2 Fm/block $/ C_{\max } / d_{i}: \quad \min C_{\max } \equiv \min C_{m, T}(10)$
Sujeto a:

$\begin{array}{lll}\sum_{i=1}^{n} x_{i, t}=1 & \forall t=1, . ., T & \\ \sum_{t=1}^{T} x_{i, t}=d_{i} & \forall i=1, . ., n & \\ \rho_{k, t}=\sum_{i=1}^{n} p_{i, k} x_{i, t} & \forall k=1, ., m & \forall t=1, . ., T \\ C_{k, t} \geq C_{k, t-1}+\rho_{k, t} & \forall k=1, ., m & \forall t=1, . ., T \\ C_{k, t} \geq C_{k-1, t}+\rho_{k, t} & \forall k=1, ., m & \forall t=1, . ., T \\ C_{k, t} \geq C_{k+1, t-1} & \forall k=1, ., m & \forall t=1, . ., T \\ X_{i, t} \in\{0,1\} & \forall i=1, ., n & \\ C_{k, 0}=0 & \forall k=1, ., m & \\ C_{0, t}=0 & \forall t=1, . ., T & \\ C_{m+1, t}=0 & \forall t=1, . ., T & \end{array}$

En el modelo MILP-2, obviamente tanto la función objetivo (10) como las restricciones (11) a (15) y (17) a (19) son consecutivamente coincidentes con las fórmulas (1) a (9) del modelo MILP-1. Los cambios que se añaden al considerar posibles bloqueos entre máquinas son:

- $\quad$ Aquí $\mathrm{C}_{\mathrm{k}, \mathrm{t}}$ representa el instante de liberación (frente al de compleción) del t-ésimo trabajo $\pi_{t}$ de la secuencia de fabricación $\pi(\mathrm{T})$ en la máquina $\mathrm{k} \in \mathrm{K}$.

- $\quad$ Las restricciones (16) sirven para acotar inferiormente el instante de liberación $C_{k, t}$ con el instante de liberación del trabajo anterior $\left(\pi_{\mathrm{t}-1}\right)$ en la máquina siguiente $(\mathrm{k}+1)$.

- $\quad$ Las igualdades (20) son, por convenio, las inicializaciones de los instantes de liberación en la máquina virtual: $\mathrm{k}=\mathrm{m}+1$.

\section{Caso de estudio en la factoría Nissan de Barcelona}

\subsection{Descripción del caso}

Una línea de ensamblado de la planta de motores de Nissan Spanish Industrial Operations en Barcelona está constituida por 21 estaciones de trabajo $(\mathrm{k}=1, ., 21)$, estando capacitada para fabricar 270 motores diarios en dos turnos de trabajo de unas 7 horas efectivas cada uno.

Los motores en cuestión no son idénticos, existiendo 9 tipos (i=1,.,9), los cuales se agrupan en tres familias: SUV $(i=1,2,3)$, Van $(i=4,5)$ y Truck $(i=6,7,8,9)$. En la Figura 1 se muestra el motor tipo M1 perteneciente a la familia SUV. 
Figura 1 Motor Nissan Pathfinder. Características: (i) 747 piezas y 330 referencias, (ii) 378 tareas elementales de montaje agrupadas en 140 tareas de línea de producción

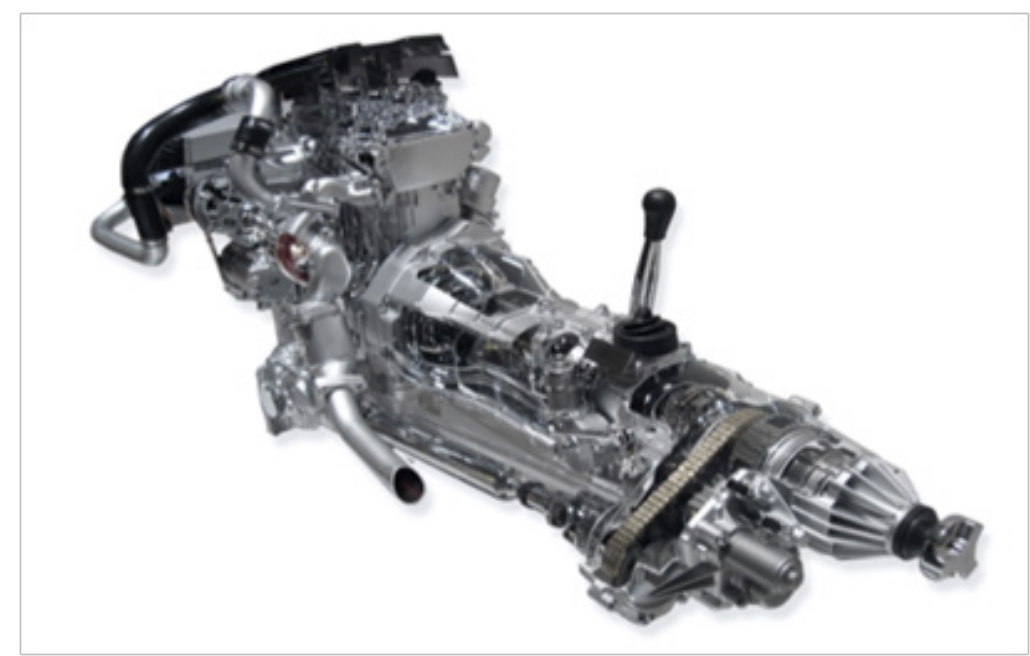

Los tiempos de proceso de las operaciones que conforman un motor, medidos en segundos a actividad normal de los operadores, son heterogéneos y oscilan entre 89 y 185 segundos con una media de 142,40 segundos y una desviación tipo de 31,14 (Bautista and Cano 2011). En la Tabla 1 se recogen dichos tiempos para cada tipo de motor $(i=1, ., 9)$ y cada puesto de trabajo $(\mathrm{k}=1, ., 21)$. 
Tabla 1 Tiempos de proceso a actividad normal $\left(\mathrm{p}_{\mathrm{i}, \mathrm{k}}\right)$ en segundos de los 9

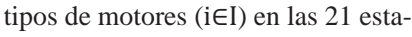
ciones de trabajo $(\mathrm{k} \in \mathrm{K})$ del conjunto de instancias Nissan-9Ing.I (Bautista and Cano 2011)

\begin{tabular}{|c|c|c|c|c|c|c|c|c|c|}
\hline $\mathbf{k} \backslash \mathbf{i}$ & M1 & M2 & M3 & M4 & M5 & M6 & M7 & M8 & M9 \\
\hline 1 & 104 & 100 & 97 & 92 & 100 & 94 & 103 & 109 & 101 \\
\hline 2 & 103 & 103 & 105 & 107 & 101 & 108 & 106 & 102 & 110 \\
\hline 3 & 165 & 156 & 164 & 161 & 148 & 156 & 154 & 164 & 155 \\
\hline 4 & 166 & 175 & 172 & 167 & 168 & 167 & 168 & 156 & 173 \\
\hline 5 & 111 & 114 & 114 & 115 & 117 & 117 & 115 & 111 & 111 \\
\hline 6 & 126 & 121 & 122 & 124 & 127 & 130 & 120 & 121 & 134 \\
\hline 7 & 97 & 96 & 96 & 93 & 96 & 89 & 94 & 101 & 92 \\
\hline 8 & 100 & 97 & 95 & 106 & 94 & 102 & 103 & 102 & 100 \\
\hline 9 & 179 & 174 & 173 & 178 & 178 & 171 & 177 & 171 & 174 \\
\hline 10 & 178 & 172 & 172 & 177 & 178 & 177 & 175 & 173 & 175 \\
\hline 11 & 161 & 152 & 168 & 167 & 167 & 166 & 172 & 157 & 177 \\
\hline 12 & 96 & 106 & 105 & 97 & 101 & 100 & 96 & 104 & 96 \\
\hline 13 & 99 & 101 & 102 & 101 & 99 & 101 & 96 & 102 & 99 \\
\hline 14 & 147 & 155 & 142 & 154 & 146 & 143 & 154 & 153 & 155 \\
\hline 15 & 163 & 152 & 156 & 152 & 153 & 152 & 154 & 156 & 156 \\
\hline 16 & 163 & 185 & 183 & 178 & 169 & 173 & 172 & 182 & 171 \\
\hline 17 & 173 & 179 & 178 & 169 & 173 & 178 & 174 & 175 & 175 \\
\hline 18 & 176 & 167 & 181 & 180 & 172 & 173 & 173 & 168 & 184 \\
\hline 19 & 162 & 150 & 152 & 152 & 160 & 151 & 155 & 148 & 167 \\
\hline 20 & 164 & 161 & 157 & 159 & 162 & 160 & 162 & 158 & 157 \\
\hline 21 & 177 & 161 & 154 & 168 & 172 & 170 & 167 & 149 & 169 \\
\hline Mínimo & 96 & 96 & 95 & 92 & 94 & 89 & 94 & 101 & 92 \\
\hline Máximo & 179 & 185 & 183 & 180 & 178 & 178 & 177 & 182 & 184 \\
\hline Media & 143,33 & 141,76 & 142,29 & 142,71 & 141,95 & 141,81 & 142,38 & 141,05 & 144,33 \\
\hline
\end{tabular}


El sistema productivo original es una línea de modelos mixtos con estaciones cerradas en la que cada estación $(\mathrm{k}=1, ., 21)$ dispone de un tiempo de ciclo de 175 segundos para realizar cada operación.

Estrictamente, la línea se puede considerar semicerrada (semiabierta, si se prefiere), ya que los operadores de cada estación de trabajo (operarios y robots) disponen de un tiempo adicional de 20 segundos como máximo, llamado ventana temporal, para completar una operación, si ello fuera necesario. No obstante, la utilización del tiempo extra en una estación concreta y en un momento determinado implica la reducción del tiempo disponible en las estaciones siguientes en el futuro (Bautista and Cano 2011). Atendiendo a las barrreras marcadas por tiempo de ciclo y la ventana temporal, cuando no es posible concluir una operación, entonces, queda pendiente un trabajo que deberá completarse en una línea de recuperación que se activará para paliar tales efectos; la otra alternativa, menos recomendable, es parar la línea hasta que se haya completado el trabajo.

Consecuentemente, el tiempo que dispone la línea para concluir todas las operaciones de un plan de 270 motores es igual a 50750 segundos, a los que cabe añadir 20 segundos más si se contempla la gentileza de permitir a la última estación de trabajo el uso de la ventana temporal con la última unidad.

Las preguntas que formulamos son: ¿ofrece alguna ventaja competitiva un taller de flujo regular frente a una línea de montaje de modelos mixtos? ¿qué impacto económico genera la supresión de espacios entre estaciones consecutivas?

El nuevo entorno de fabricación que queremos considerar en este estudio es un taller de flujo regular orientado al producto. Y se desea establecer secuencias de producción de 270 motores en total, compuestas por motores de los 9 tipos, atendiendo a diversos planes de demanda.
Por consiguiente, las secuencias de fabricación de motores deben cumplir con el objetivo de mínimo $\mathrm{C}_{\max }$, $\mathrm{y}$ deben tener en cuenta dos configuraciones de producción en taller de flujo regular:

- $\quad$ Taller T1: Espacio de almacenamiento ilimitado entre las parejas de estaciones de trabajo consecutivas (problema $\mathrm{Fm} / \mathrm{prmu} / \mathrm{C}_{\max }$ ). Esto equivale a poder apartar los motores del flujo de producción cuando concluye la operación en cada estación.

- $\quad$ Taller T2: Espacio de almacenamiento intermedio suprimido. Existe, por tanto, el posible bloqueo entre estaciones (problema Fm/block/ $\mathrm{C}_{\max }$ ).

El primer objetivo de este caso es analizar el impacto económico generado por el cambio de sistema productivo cuando se pasa de una línea de ensamblado clásica con estaciones cerradas (caso original) a un entorno de fabricación tipo taller mecánico (Flow Shop) de flujo regular. Para ello, recurriremos a los modelos $\mathrm{Fm} / \beta / \gamma / \mathrm{d}_{\mathrm{i}}$.

El segundo objetivo es medir el impacto, tanto económico como computacional, que genera la variación de la demanda en los sistemas productivos objeto de estudio.

Una alternativa para tratar la variabilidad de la demanda es recurrir a diversos escenarios. Los escenarios deben ser realistas estando en sintonía con la capacidad productiva y el histórico de la planta de fabricación. Formalmente agruparemos los escenarios en un conjunto de planes E de elementos $\varepsilon \in \mathrm{E}$.

De manera similar a trabajos anteriores, consideraremos 23 planes de producción para los 9 tipos de motores y un día de trabajo (dos turnos). Cada plan da lugar a unas cargas de las estaciones de trabajo que se determinan en función de la demandas de los tipos de motor.

En la Tabla 2 se muestran los 23 planes mencionados cuyas demandas totales son idéntica e iguales a 270 motores. 
Tabla 2 Demandas diarias por producto y plan $\left(\mathrm{d}_{\mathrm{i}, \varepsilon}\right)$ para las 23 instancias Nissan-9Eng.I $(\varepsilon \in \mathrm{E})$. Los planes se han agrupado en 7 categorías. Cada categoría refleja una situación representativa de la variabilidad de la demanda que afecta a las cargas de trabajo en las estaciones.

\begin{tabular}{|c|c|c|c|c|c|c|c|c|c|c|c|c|c|}
\hline$\varepsilon \in \mathrm{E}$ & M1 & M2 & M3 & M4 & M5 & M6 & M7 & M8 & M9 & SUV & Van & Truck & Total \\
\hline 1 & 30 & 30 & 30 & 30 & 30 & 30 & 30 & 30 & 30 & 90 & 60 & 120 & 270 \\
\hline 2 & 30 & 30 & 30 & 45 & 45 & 23 & 23 & 22 & 22 & 90 & 90 & 90 & 270 \\
\hline 3 & 10 & 10 & 10 & 60 & 60 & 30 & 30 & 30 & 30 & 30 & 120 & 120 & 270 \\
\hline 4 & 40 & 40 & 40 & 15 & 15 & 30 & 30 & 30 & 30 & 120 & 30 & 120 & 270 \\
\hline 5 & 40 & 40 & 40 & 60 & 60 & 8 & 8 & 7 & 7 & 120 & 120 & 30 & 270 \\
\hline 6 & 50 & 50 & 50 & 30 & 30 & 15 & 15 & 15 & 15 & 150 & 60 & 60 & 270 \\
\hline 7 & 20 & 20 & 20 & 75 & 75 & 15 & 15 & 15 & 15 & 60 & 150 & 60 & 270 \\
\hline 8 & 20 & 20 & 20 & 30 & 30 & 38 & 38 & 37 & 37 & 60 & 60 & 150 & 270 \\
\hline 9 & 70 & 70 & 70 & 15 & 15 & 8 & 8 & 7 & 7 & 210 & 30 & 30 & 270 \\
\hline 10 & 10 & 10 & 10 & 105 & 105 & 8 & 8 & 7 & 7 & 30 & 210 & 30 & 270 \\
\hline 11 & 10 & 10 & 10 & 15 & 15 & 53 & 53 & 52 & 52 & 30 & 30 & 210 & 270 \\
\hline 12 & 24 & 23 & 23 & 45 & 45 & 28 & 28 & 27 & 27 & 70 & 90 & 110 & 270 \\
\hline 13 & 37 & 37 & 36 & 35 & 35 & 23 & 23 & 22 & 22 & 110 & 70 & 90 & 270 \\
\hline 14 & 37 & 37 & 36 & 45 & 45 & 18 & 18 & 17 & 17 & 110 & 90 & 70 & 270 \\
\hline 15 & 24 & 23 & 23 & 55 & 55 & 23 & 23 & 22 & 22 & 70 & 110 & 90 & 270 \\
\hline 16 & 30 & 30 & 30 & 35 & 35 & 28 & 28 & 27 & 27 & 90 & 70 & 110 & 270 \\
\hline 17 & 30 & 30 & 30 & 55 & 55 & 18 & 18 & 17 & 17 & 90 & 110 & 70 & 270 \\
\hline 18 & 60 & 60 & 60 & 30 & 30 & 8 & 8 & 7 & 7 & 180 & 60 & 30 & 270 \\
\hline 19 & 10 & 10 & 10 & 90 & 90 & 15 & 15 & 15 & 15 & 30 & 180 & 60 & 270 \\
\hline 20 & 20 & 20 & 20 & 15 & 15 & 45 & 45 & 45 & 45 & 60 & 30 & 180 & 270 \\
\hline 21 & 60 & 60 & 60 & 15 & 15 & 15 & 15 & 15 & 15 & 180 & 30 & 60 & 270 \\
\hline 22 & 20 & 20 & 20 & 90 & 90 & 8 & 8 & 7 & 7 & 60 & 180 & 30 & 270 \\
\hline 23 & 10 & 10 & 10 & 30 & 30 & 45 & 45 & 45 & 45 & 30 & 60 & 180 & 270 \\
\hline
\end{tabular}


Los planes de la Tabla 2 se han agrupado en 7 categorías, en función de la dispersión de las demandas entre las tres familias de motores. Dichas categorías son:

- $\quad$ Categoría-1 (plan \#1): Plan con demanda equilibrada, es decir, idéntica demanda para cada uno de los 9 tipos de motor: 30 motores de cada tipo. Posiblemente, esta categoría es la menos realista, aunque es útil como plan referente a la hora de analizar la dispersión del tiempo de compleción (makespan) de todas las operaciones.

- $\quad$ Categoría-2 (plan \#2): Plan con idéntica demanda para cada familia de motores: SUV, Van y Truck (90 unidades por familia de producto).

- $\quad$ Categoría-3 (planes \#3, \#4 y \#5): Una familia con baja demanda, mientras que las otras dos familias presentan alta demanda e idéntica.

- $\quad$ Categoría-4 (planes \#6, \#7 y \#8): Una familia con alta demanda, mientras que las otras dos familias presentan demandas medias e idénticas.

- $\quad$ Categoría-5 (planes \#9, \#10 y \#11): Una familia con alta demanda, mientras que las otras dos familias presentan demandas bajas e idénticas.

- $\quad$ Categoría-6 (planes \#12 a \#17): Demandas de las familias en progresión aritmética.

- $\quad$ Categoría-7 (planes \#18 a \#17): Demandas de las familias en progresión no aritmética.

Atendiendo a las 7 categorías propuestas, cabe esperar un comportamiento de la Programación Lineal Entera Mixta (MILP) diverso en cuanto a la calidad de las soluciones ofrecidas y los tiempos de CPU requeridos para el conjunto de instancias Nissan-9Eng.I.

\subsection{Procedimientos}

Los códigos compilados de los procedimiento han sido ejecutados en un ordenador DELL Inspiron-13 (Intel(R) Core(TM) i7-7500U CPU @ 2.70 GHz 2.90 GHz, 16 GB de RAM, x64 Windows 10 Pro). Las características de los 2 procedimientos son:

MILP-1: Modelo Fm/prmu/C $\mathrm{max}_{\max } / \mathrm{d}_{\mathrm{i}}$ : (i) Función objetivo con el propósito de minimizar el valor de $\mathrm{C}_{\max }$ de la secuencia de fabricación; (ii) implementación para solver IBM ILOG CPLEX (Optimization Studio v.12.2, win-x86-64); (iii) tiempo de CPU máximo concedido para resolver cada instancia igual a 180 s. (23 instancias: Nissan-9Eng.I).
- $\quad$ MILP-2: Modelo Fm/block/ $\mathrm{C}_{\max } / \mathrm{d}_{\mathrm{i}}$ : (i) Función objetivo con el propósito de minimizar el valor de $\mathrm{C}_{\max }$ de la secuencia de fabricación; (ii) implementación para solver IBM ILOG CPLEX (Optimization Studio v.12.2, win-x86-64); (iii) tiempo de CPU máximo concedido para resolver cada instancia igual a 180 s. (23 instancias: Nissan-9Eng.I).

\subsection{Resultados}

Acto seguido, procedemos a aplicar los modelos MILP1 y MILP-2, correspondientes a los problemas Fm/prmu/ $\mathrm{C}_{\max } / \mathrm{d}_{\mathrm{i}}$ y Fm/block/ $\mathrm{C}_{\max } / \mathrm{d}_{\mathrm{i}}$, al conjunto de instancias Nissan-9Eng.I, utilizando el solucionador CPLEX.

Para analizar y comentar los resultados emplearemos los siguientes atributos:

$\varepsilon \in E$ Número identificativo de la instancia del conjunto Nissan-9Eng.I.

$\mathrm{C}_{\max }$ Mejor valor del makespan obtenido por los procedimientos MILP-1 o MILP-2

$\mathrm{C}_{\text {max }}^{*}$ Valor óptimo del makespan.

$\mathrm{C}^{0}{ }_{\text {max }}$ Mejor valor conocido del makespan. Ante la ausencia de valores en entorno de taller de flujo regular para el conjunto de instancias Nissan-9Eng.I, en este trabajo consideramos como mejor valor conocido el tiempo disponible para realizar todos los trabajos en la línea de montaje original: $\mathrm{C}_{\max }^{0}=50770$.

$\Delta \mathrm{C}_{\max }$ Incremento de $\mathrm{C}_{\max }$, esto es: $\Delta \mathrm{C}_{\max }=\mathrm{C}_{\max }-\mathrm{C}_{\text {max }}^{0}$

$\mathrm{LB}_{\mathrm{p}}$ Cota inferior de $\mathrm{C}_{\max }$ para el problema Fm/prmu/ $\mathrm{C}_{\text {max }} /$ $\mathrm{d}_{\mathrm{i}}$ obtenida por MILP. Los valores de $\mathrm{LB}_{\mathrm{p}}$ corresponden al problema $\mathrm{Fm} / \mathrm{prmu} / \mathrm{C}_{\max } / \mathrm{d}_{\mathrm{i}}$ y se obtienen directamente con el procedimiento MILP-1.

$\mathrm{LB}_{\mathrm{b}}$ Cota inferior de $\mathrm{C}_{\max }$ para el problema Fm/block/ $\mathrm{C}_{\max } /$ $\mathrm{d}_{\mathrm{i}}$ obtenida por MILP-1 o MILP-2. Los valores de LB $_{\mathrm{b}}$ son el máximo entre $\mathrm{LB}_{\mathrm{p}} \mathrm{y}$ la cota inferior ofrecida por el procedimiento MILP-2 para el problema Fm/block/ $\mathrm{C}_{\max } / \mathrm{d}_{\mathrm{i}}$.

Gap $_{\mathrm{C}^{*}} \quad$ Gap relativo entre $\mathrm{C}_{\max } \mathrm{y} \mathrm{C}_{\text {max }}^{*}$

Gap $_{\mathrm{C}^{0}} \quad$ Gap relativo entre $\mathrm{C}_{\text {max }} \mathrm{y} \mathrm{C}_{\text {max }}^{0}$

Gap $_{\text {LB_p }} \quad$ Gap relativo entre $\mathrm{C}_{\text {max }}$ y LB

Gap $_{\text {LB_b }} \quad$ Gap relativo entre $C_{\text {max }}$ y LB 
$\varphi \quad$ Coste por caída de producción. Se valora en 137,14 euros por minuto productivo.

$\mathrm{G}(\bullet), \mathrm{C}(\bullet)$ Ganancia diaria y Coste (Pérdida) diario medidos en euros $(€)$ o en motores $(\mathrm{m})$.

Los valores del Gap relativo entre $C_{\max }$ y el resto de atributos relacionados con el makespan $\left(\mathrm{C}^{*}{ }_{\max }, \mathrm{C}^{0}{ }_{\max }, \mathrm{LB}_{\mathrm{p}}, \mathrm{LB}_{\mathrm{b}}\right)$ se determinan según la fórmula (21).

$\operatorname{Gap}_{X}(\varepsilon)=\frac{C_{\max }(\varepsilon)-X(\varepsilon)}{C_{\max }(\varepsilon)} \mathrm{X} \in\left\{\mathrm{LB}_{\mathrm{p}}, \mathrm{LB}_{\mathrm{b}^{\prime}} \mathrm{C}^{*}{ }_{\text {max }}, \mathrm{C}^{0}{ }_{\max }\right\}, \forall \varepsilon \in \mathrm{E}, \forall \varepsilon \in \mathrm{E}^{\prime}(21)$
Los valores de $C_{\text {max }}^{*}$ son óptimos confirmados en este trabajo con el procedimiento MILP-1.

Por su parte, el coste por caída de producción $\varphi$ (ver Bautista et al. 2018) se obtiene teniendo en cuenta que el valor promedio de un motor es de $4000 €$ y que el valor añadido por la línea al producto es del $10 \%$.

En tales condiciones, en la Tabla 3 se muestran los resultados del experimento conseguidos por CPLEX para el modelo Fm/prmu/C $\mathrm{C}_{\max } / \mathrm{d}_{\mathrm{i}}$.
Tabla 3 Resultados de MILP-1 para el problema $\mathrm{Fm} /$ prmu/ $\mathrm{C}_{\max } / \mathrm{d}_{\mathrm{i}}$ sobre las instancias Nissan-9Eng.I ( $\varepsilon \in E$ ). Los valores $\mathrm{C}^{*}{ }_{\text {max }}$ son óptimos confirmados (ver valores de $\mathrm{LB}_{\mathrm{p}}$ ). Los valores del $\mathrm{Gap}_{\text {Cmax }}^{0}$ son todos negativos, indicando que el taller de flujo regular propicia una reducción de $\mathrm{C}_{\max }$ respecto a la línea de montaje original.

\begin{tabular}{|c|c|c|c|c|c|c|}
\hline$\varepsilon \in \mathrm{E}$ & $\mathrm{C}_{\text {max }}^{*}$ & $\mathrm{LB}_{\mathrm{p}}$ & $\Delta \mathrm{C}_{\text {max }}$ & $\mathrm{Gap}^{0}{ }_{\mathrm{C} \text { max }}$ & $\mathrm{G}(€)$ & CPU (s) \\
\hline 1 & 50091 & 50091 & $-679,00$ & $-0,014$ & 1552,00 & 45,84 \\
\hline 2 & 50174 & 50174 & $-596,00$ & $-0,012$ & 1362,29 & 15,19 \\
\hline 3 & 50301 & 50301 & $-469,00$ & $-0,009$ & 1072,00 & 10,34 \\
\hline 4 & 50167 & 50167 & $-603,00$ & $-0,012$ & 1378,29 & 13,61 \\
\hline 5 & 50379 & 50379 & $-391,00$ & $-0,008$ & 893,71 & 9,85 \\
\hline 6 & 50202 & 50202 & $-568,00$ & $-0,011$ & 1298,29 & 14,26 \\
\hline 7 & 50395 & 50395 & $-375,00$ & $-0,007$ & 857,14 & 8,29 \\
\hline 8 & 50123 & 50123 & $-647,00$ & $-0,013$ & 1478,86 & 12,42 \\
\hline 9 & 50378 & 50378 & $-392,00$ & $-0,008$ & 896,00 & 10,39 \\
\hline 10 & 50619 & 50619 & $-151,00$ & $-0,003$ & 345,14 & 7,62 \\
\hline 11 & 50078 & 50078 & $-692,00$ & $-0,014$ & 1581,71 & 25,30 \\
\hline 12 & 50192 & 50192 & $-578,00$ & $-0,012$ & 1321,14 & 17,41 \\
\hline 13 & 50123 & 50123 & $-647,00$ & $-0,013$ & 1478,86 & 14,81 \\
\hline 14 & 50218 & 50218 & $-552,00$ & $-0,011$ & 1261,71 & 10,07 \\
\hline 15 & 50242 & 50242 & $-528,00$ & $-0,011$ & 1206,86 & 10,51 \\
\hline 16 & 50118 & 50118 & $-652,00$ & $-0,013$ & 1490,29 & 55,80 \\
\hline 17 & 50269 & 50269 & $-501,00$ & $-0,010$ & 1145,14 & 10,59 \\
\hline 18 & 50273 & 50273 & $-497,00$ & $-0,010$ & 1136,00 & 14,28 \\
\hline 19 & 50475 & 50475 & $-295,00$ & $-0,006$ & 674,29 & 8,09 \\
\hline 20 & 50089 & 50089 & $-681,00$ & $-0,014$ & 1556,57 & 96,14 \\
\hline 21 & 50307 & 50307 & $-463,00$ & $-0,009$ & 1058,29 & 13,77 \\
\hline
\end{tabular}




\begin{tabular}{ccccccc}
\hline 22 & 50539 & 50539 & $-231,00$ & $-0,005$ & 528,00 & 7,31 \\
\hline 23 & 50151 & 50151 & $-619,00$ & $-0,012$ & 1414,86 & 13,67 \\
\hline Media & 50256,65 & 50256,65 & $-513,35$ & $-0,010$ & 1173,37 & 19,37 \\
\hline Max & & & & & \\
\hline Min & 50619 & 50619 & $-151,00$ & $-0,003$ & 1581,71 & 96,14 \\
\hline
\end{tabular}

Por su parte, en la Tabla 4 se recogen los resultados para el modelo Fm/block/ $\mathrm{C}_{\max } / \mathrm{d}_{\mathrm{i}}$ con posibilidad de bloqueo entre estaciones que se obtienen mediante el solucionador CPLEX
Tabla 4 Resultados de MILP-2 para el problema Fm/block $/ \mathrm{C}_{\max } / \mathrm{d}_{\mathrm{i}}$ sobre las instancias Nissan-9Eng.I $(\varepsilon \in \mathrm{E})$. No se confirma ningún óptimo para $\mathrm{C}_{\text {max }}$ (comparar valores con $\mathrm{LB}_{\mathrm{b}}$ ). Los valores del $\mathrm{Gap}^{0}{ }_{\text {Cmax }}$ positivos indican que el taller de flujo regular propicia el incremento de $\mathrm{C}_{\max }$ respecto a la línea de montaje original.

\begin{tabular}{|c|c|c|c|c|c|c|c|}
\hline$\varepsilon \in \mathrm{E}$ & $\mathrm{C}_{\max }$ & $\mathrm{LB}_{\mathrm{b}}$ & $\Delta \mathrm{C}_{\text {max }}$ & Gapp $^{0}{ }_{\text {max }}$ & $\operatorname{Gap}_{\mathrm{LB} b \mathrm{~b}}$ & $C(€)$ & $\mathrm{CPU}(\mathrm{s})$ \\
\hline 1 & 51094 & 50091 & 324,00 & 0,006 & 0,020 & 740,57 & 180,33 \\
\hline 2 & 51006 & 50174 & 236,00 & 0,005 & 0,016 & 539,43 & 180,25 \\
\hline 3 & 50757 & 50301 & $-13,00$ & 0,000 & 0,009 & $-29,71$ & 180,13 \\
\hline 4 & 51310 & 50167 & 540,00 & 0,011 & 0,022 & 1234,29 & 180,25 \\
\hline 5 & 50972 & 50379 & 202,00 & 0,004 & 0,012 & 461,71 & 180,10 \\
\hline 6 & 51072 & 50203 & 302,00 & 0,006 & 0,017 & 690,29 & 180,17 \\
\hline 7 & 50713 & 50395 & $-57,00$ & $-0,001$ & 0,006 & $-130,29$ & 180,14 \\
\hline 8 & 51167 & 50123 & 397,00 & 0,008 & 0,020 & 907,43 & 180,15 \\
\hline 9 & 51385 & 50378 & 615,00 & 0,012 & 0,020 & 1405,71 & 180,15 \\
\hline 10 & 50671 & 50620 & $-99,00$ & $-0,002$ & 0,001 & $-226,29$ & 180,24 \\
\hline 11 & 50933 & 50078 & 163,00 & 0,003 & 0,017 & 372,57 & 180,19 \\
\hline 12 & 51071 & 50193 & 301,00 & 0,006 & 0,017 & 688,00 & 180,16 \\
\hline 13 & 51125 & 50124 & 355,00 & 0,007 & 0,020 & 811,43 & 180,19 \\
\hline 14 & 51066 & 50219 & 296,00 & 0,006 & 0,017 & 676,57 & 180,19 \\
\hline 15 & 50967 & 50242 & 197,00 & 0,004 & 0,014 & 450,29 & 180,14 \\
\hline 16 & 51104 & 50118 & 334,00 & 0,007 & 0,019 & 763,43 & 180,13 \\
\hline 17 & 50951 & 50270 & 181,00 & 0,004 & 0,013 & 413,71 & 180,14 \\
\hline 18 & 51267 & 50273 & 497,00 & 0,010 & 0,019 & 1136,00 & 180,16 \\
\hline 19 & 50648 & 50476 & $-122,00$ & $-0,002$ & 0,003 & $-278,86$ & 180,16 \\
\hline 20 & 51112 & 50092 & 342,00 & 0,007 & 0,020 & 781,71 & 180,14 \\
\hline
\end{tabular}




\begin{tabular}{|c|c|c|c|c|c|c|c|}
\hline 21 & 51385 & 50307 & 615,00 & 0,012 & 0,021 & 1405,71 & 180,17 \\
\hline 22 & 50690 & 50540 & $-80,00$ & $-0,002$ & 0,003 & $-182,86$ & 180,13 \\
\hline 23 & 50920 & 50151 & 150,00 & 0,003 & 0,015 & 342,86 & 180,18 \\
\hline Media & 51016,78 & 50257,13 & 246,78 & 0,005 & 0,015 & 564,07 & 180,17 \\
\hline Max & 51385 & 50620 & 615,00 & 0,012 & 0,022 & 1405,71 & 180,33 \\
\hline Min & 50648 & 50078 & $-122,00$ & $-0,002$ & 0,001 & $-278,86$ & 180,10 \\
\hline
\end{tabular}

A La vista de la Tabla 3 podemos extraer las siguientes conclusiones:

- $\quad$ El procedimiento MILP-1 obtiene y confirma las soluciones óptimas para las 23 instancias del conjunto Nissan-9Eng.I cuando resolvemos el problema $\mathrm{Fm} / \mathrm{prmu} / \mathrm{C}_{\max } / \mathrm{d}_{\mathrm{i}}$.

- $\quad$ El tiempo medio de CPU empleado por MILP-1 es aproximadamente 19,37 segundos para cada instancia con 270 trabajos (motores) agrupados en 9 tipos. El caso más favorable con 7,31 segundos corresponde a la instancia \#22, mientras que el peor caso con 96,14 segundos corresponde a la instancia \#20. Ambas instancias pertenecen a la categoría 7 (demandas de familias en progresión no aritmética).

- $\quad$ La reducción del tiempo de compleción, $-\Delta \mathrm{C}_{\max }$, al transformar la línea de montaje original en un taller de flujo regular es de 513,35 segundos en promedio, estando tales reducciones en un rango de valores comprendido entre 151 y 692, correspondiendo a las instancias \#10 y \#11 de la categoría 5, respectivamente. En caso de no aplicar dicho ahorro a la reducción de la jornada laboral, esto permitiría fabricar en promedio 2,93 motores más al día.

- $\quad$ La ganancia económica diaria $\left(\mathrm{G}(€)=-\varphi \Delta \mathrm{C}_{\max }\right)$ debida a la reducción del tiempo total de compleción durante los dos turnos de trabajo, presenta un valor medio de $1173,37 €$ y un rango comprendido entre $345,14 €$ y $1581,71 €$.

Teniendo en cuenta los comentarios anteriores, podemos concluir que no existe razón suficiente para descartar la programación lineal entera mixta como un procedimiento garante de buenas soluciones para el problema $\mathrm{Fm} / \mathrm{prmu} / \mathrm{C}_{\max } / \mathrm{d}_{\mathrm{i}}$ frente a instancias de dimensiones industriales en el sector de automoción.

De manera similar, atendiendo ahora a la Tabla 4 podemos extraer otras conclusiones para el caso en que no hay espacio entre estaciones para el almacenamiento intermedio.
Las conclusiones, algunas de ellas en discordia con las primeras, son las siguientes:

- El procedimiento MILP-2 no confirma ninguna solución óptima del conjunto de instancias Nissan-9Eng.I cuando resolvemos el problema Fm/block/ $\mathrm{C}_{\max } / \mathrm{d}_{\mathrm{i}}$ y limitamos el tiempo de CPU del solucionador CPLEX a 3 minutos. Por ello, el tiempo medio de CPU empleado por MILP-2 que aparece en la tabla es de 180,17 segundos por instancia.

- $\quad$ El incremento del tiempo de compleción, $\Delta \mathrm{C}_{\max }$, es de 246,78 segundos en promedio con un rango de valores limitado por el intervalo [-122; 615], existiendo valores negativos para $\Delta \mathrm{C}_{\max }$, por lo que se abre la posibilidad de aumentar la productividad del sistema cuando se den los correspondientes planes de demanda. No obstante, la tónica general de los resultados ofrecidos por MILP-2 es que la eliminación de espacios entre estaciones provoca caídas de producción, las cuales equivalen a fabricar 1,41 motores menos al día en promedio, pudiendo llegar en el peor de los casos a 3,51 motores al día (instancia \#9 de la categoría 5).

- La calidad de las soluciones ofrecidas por CPLEX (180s. CPU) la medimos con el atributo Gap LB_b, $_{\text {, }}$ obteniendo un valor promedio del $1,49 \%$ respecto a la mejor cota utilizada, siendo el mejor resultado para este atributo el que se alcanza en la instancia \#10 con el $0,1 \%$ y el peor valor el de la instancia \#4 con el 2,23\%. Esto significa que existe margen para mejorar las soluciones.

- $\quad$ El coste diario por caída de producción $\left(C(€)=\varphi \Delta C_{\max }\right)$, durante los dos turnos de trabajo, presenta un valor medio de 564,07€ con un rango de costes que está limitado por el intervalo [-278,86; 1405,71]; esto supone que el máximo ahorro (coste negativo) se consigue en la instancia \#19, mientras que la mayor pérdida corresponde a la instancia \#9.

Desde el punto de vista computacional, aquí también es válido el principio laplaciano que nos lleva a afirmar que no existe razón suficiente para descartar la programación lineal 
entera mixta como método para resolver instancias de tamaño industrial en el sector de automoción relativas al problema de secuenciación Fm/block/ $\mathrm{C}_{\max } / \mathrm{d}_{\mathrm{i}}$. De hecho, se han resuelto modelos MILP que han requerido 14850 variables (2430 de ellas binarias) y 25682 restricciones, concediendo a CPLEX un tiempo de CPU de 3 minutos, en un ordenador DELL Inspiron-13, y garantizando soluciones que están en promedio al $1,49 \%$ de la mejor cota. No obstante, aun reconociendo que el margen no es excesivo, cabe decir que es posible mejorar los resultados en el conjunto Nissan-9Eng.I empleando más tiempo de computación con CPLEX o con otros procedimientos exactos y heurísticos.

Por otra parte, comparando los resultados ofrecidos por MILP-1 y MILP-2, queda claro que un taller de flujo regular con espacio ilimitado entre estaciones ofrece más ventajas desde el punto de vista productivo que un taller con flujo tenso sin posibilidad de almacenamientos intermedios o, incluso, que una línea de modelos mixtos.

Para ilustrar la anterior afirmación, en la Figura 2 se muestran las pérdidas y ganancias de la producción de motores, para las 23 instancias Nissan-9Eng.I, según los dos tipos de taller de flujo regular considerados: $\mathrm{Fm} / \mathrm{prmu} / \mathrm{C}_{\max } / \mathrm{d}_{\mathrm{i}}$ y Fm/ block $/ \mathrm{C}_{\max } / \mathrm{d}_{\mathrm{i}}$. La ganancia en motores (valores positivos) se calcula de la forma siguiente: $G(m)=-\Delta C_{\max } / c$, donde el parámetro c simboliza el tiempo de ciclo de la línea de montaje original, cuyo valor es de 175 segundos en este trabajo.

Figura 2 : Resultados de MILP-1 (prmu) y MILP-2 (block). Ganancias y Pérdidas de producción de motores, respecto a la línea original, para las instancias Nissan-9Eng.I, según Fm/ $\mathrm{prmu} / \mathrm{C}_{\max } / \mathrm{d}_{\mathrm{i}}$ o Fm/block/ $\mathrm{C}_{\max } / \mathrm{d}_{\mathrm{i}}$

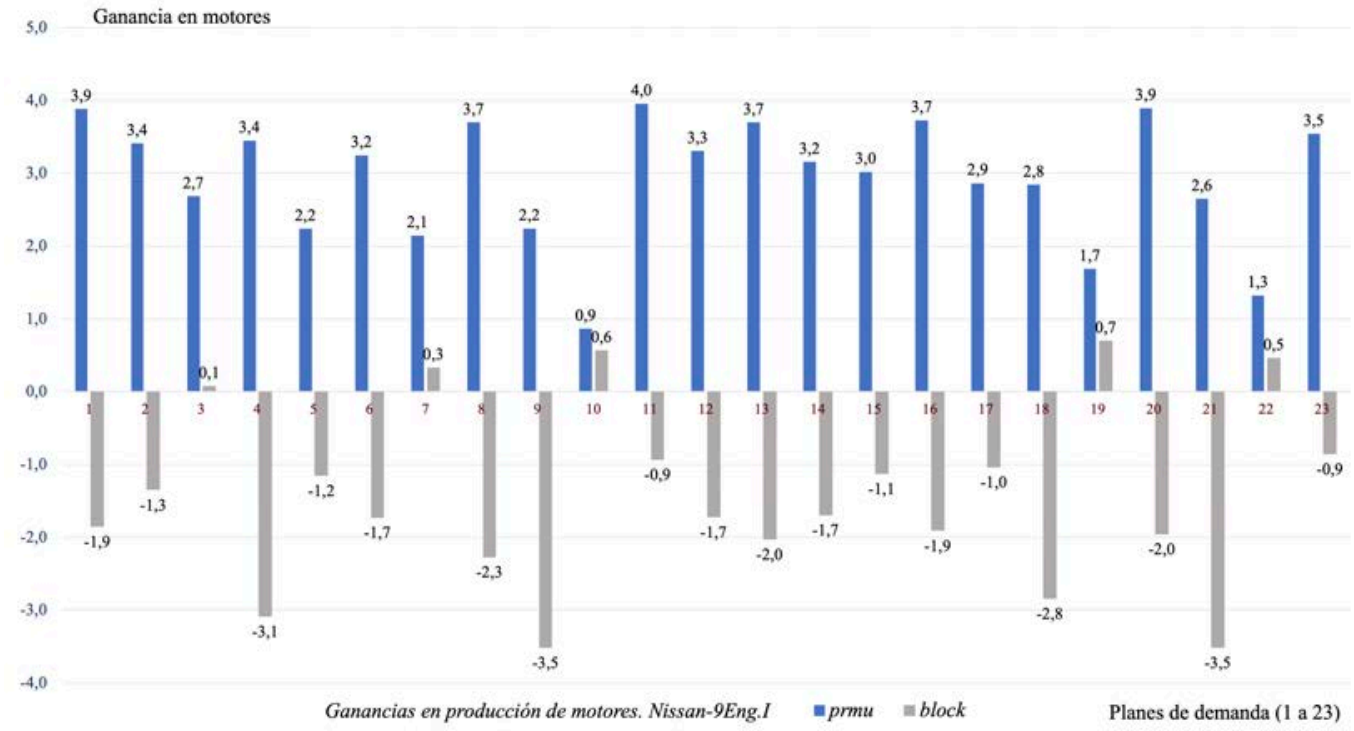

La Figura 2 permite discriminar entre los dos tipos de taller de flujo regular analizados, si se atiende a la productividad que puede ofrecer cada uno de ellos durante una jornada laboral.

En efecto, en todos los planes las soluciones de MILP-1 (prmu) corresponden a incrementos de productividad, ya que todos los valores de la ganancia $\mathrm{G}(\mathrm{m})$ son positivos. Teniendo en cuenta que la ganancia media es igual a 2,93 motores al día adicionales, el incremento de productividad medio es del 1,09\%.

Por su parte, MILP-2 (block) solo ofrece ganancias positivas en 5 de las 23 instancias (planes \#3, \#7, \#10, \#19 y \#22), en el resto de planes los resultados corresponden a pérdidas de la producción $\mathrm{C}(\mathrm{m})$ (valores negativos de $\mathrm{G}(\mathrm{m})$ ), respecto a la línea de montaje original, lo cual se traduce en una caída de la productividad del 0,52\%.

\subsection{Implantación de las soluciones. Aspectos tecnológicos de Industria 4.0}

Una vez vistas las ventajas que ofrece un taller de flujo regular frente a una línea de montaje de modelos mixtos, desde el punto de vista productivo, cabe preguntarse cómo llevar a la práctica una solución (secuencia de fabricación $\left.\pi(\mathrm{T})=\left(\pi_{1}, ., \pi_{\mathrm{T}}\right)\right)$ cuando se suprime la barrera virtual que supone marcar el ritmo de la fabricación mediante el tiempo de ciclo c.

Este hecho, aparentemente inofensivo, supone convertir los puestos de trabajo en estaciones abiertas, conduciendo tal liberación a que tanto los instantes de inicio como de finalización de las operaciones en cada estación de trabajo no se suceden periódicamente según el valor del ciclo c, sino a intervalos irregulares que dependen de la duración de cada operación y de los instantes de compleción de las operaciones en la estación en curso y en la anterior. 
En tales condiciones, para llevar a la práctica una solución, representada por una secuencia de fabricación $\pi(\mathrm{T})$, es necesario que se cumplan en general las condiciones siguientes:

C1. La solución debe ser legal. Es decir, la secuencia de fabricación $\pi(\mathrm{T})$ debe cumplir con la legalidad vigente que viene establecida por el convenio colectivo entre los trabajadores y la compaía. Este hecho significa que ningún operario podrá estar sometido a un factor de actividad por encima del valor máximo permitido legalmente, en ninguna estación $\mathrm{k} \in \mathrm{K}$ y en ningún ciclo de fabricación $t \in \mathrm{T}$, durante los dos turnos de trabajo. El cumplimiento de esta condición está garantizado cuando los tiempos de proceso $\mathrm{p}_{\mathrm{i}, \mathrm{k}}$ se miden a actividad normal, tal como sucede en nuestro caso de estudio.

C2. Los operarios deben ser compensados económicamente en función de su productividad. Pensamos que es justo compensar el sobreesfuerzo de los trabajadores cuando trabajan a un ritmo por encima de la actividad normal pactada con la compañía. Para ello, existen alternativas que permiten tal compensación (Bautista-Valhondo and Alfaro-Pozo 2018b).

C3. Los operarios deben estar adiestrados para cumplir con los requerimientos de Control de Producción. Esto significa que los trabajadores del taller de flujo regular deben conocer en todo momento la duración de la operación (a actividad normal) que deben ejecutar en su estación de trabajo, en función de la secuencia de fabricación $\pi(\mathrm{T})$.

C4. Los operarios deben estar informados sobre el ritmo y el avance de la producción en sus respectivas estaciones de trabajo. Los datos principales que debe conocer todo operario asignado a la estación de trabajo $\mathrm{k} \in \mathrm{K}$ en todo ciclo de fabricación $\mathrm{t} \in \mathrm{T}$ son:

- $\quad$ El tipo de producto i $\in$ I que llega a la estación como t-ésima unidad.

- $\quad$ El subconjunto de tareas que componen la operación en curso $(k, t)$.

- El instante de inicio de la operación en curso: $\mathrm{s}_{\mathrm{k}, \mathrm{t}}=\max \left(\mathrm{C}_{\mathrm{k}, \mathrm{t}-1}, \mathrm{C}_{\mathrm{k}-1, \mathrm{t}}\right)$; así como el instante de compleción: $\mathrm{C}_{\mathrm{k}, \mathrm{t}}=\mathrm{s}_{\mathrm{k}, \mathrm{t}}+\rho_{\mathrm{k}, \mathrm{t}^{*}}$

- $\quad$ El tiempo de proceso $\rho_{\mathrm{k}, \mathrm{t}}$ requerido para completar la operación en curso cuando ésta se realiza a actividad normal.

- El tiempo disponible para hacer la operación en curso, cuando ésta se debe realizar a una actividad distinta a la normal.
- $\quad$ Las tareas que puede dejar pendientes el operario en caso de que sea inevitable generar sobrecarga de trabajo local y temporal.

En nuestro caso de estudio, podemos afirmar:

1. El cumplimiento de la condición C1 no admite discusión en España y es una práctica común en los países miembros de la OCDE.

2. La condición C2 es fácil de conseguir mediante la negociación colectiva en los países miembros de la Unión Europea y la mayoría de los países occidentales.

3. La condición C3 está relacionada con la posibilidad de entrenar a los operarios para que adquieran nuevas habilidades. Esto es una práctica habitual en la industria de automoción de Occidente. Además, los operarios disponen de información básica sobre las operaciones y tienen tiempo suficiente para adaptar su ritmo de trabajo, pues la duración de cada operación (compuesta por 6 tareas elementales en promedio) supera los 2 minutos.

4. El cumplimiento de la condición C4 se puede conseguir con facilidad mediante el empleo de tecnologías IoT (Internet of Things) en el marco de la Industria 4.0.

En efecto, nuestra propuesta de aplicación IoT se basa en la implantación de un sistema de información asistido por tecnologías de conexión inalámbrica (WIFI) entre un ordenador de Control de Producción (v.gr. el principal) y un conjunto de tabletas personalizadas (42 tabletas para las 21 estaciones de trabajo) que ofrezcan información visual y acústica a cada operario sobre el avance de la producción en su puesto de trabajo concreto.

Con esta medida, un operario puede disponer en todo momento de la información que necesita para cada operación, recibiendo, a través de su tableta, las siguientes señales monitorizadas:

- Señal visual y aviso acústico para iniciar una operación.

- Señal visual y aviso acústico acelerado a medida que se vaya agotando el tiempo disponible para completar una operación (takt time dinámico).

- Señal visual sobre la lista dinámica de tareas pendientes de una operación durante un ciclo variable de fabricación, con la posibilidad de que el operario valide las tareas concluidas y actualice la lista de tareas en curso.

- Señal visual sobre la lista de tareas que el operario puede ignorar si es inevitable generar sobrecarga local. 


\section{Conclusiones}

En esta investigación hemos presentado y justificado una extensión natural para dos problemas de secuenciación clásicos en la literatura: $\mathrm{Fm} / \mathrm{prmu} / \mathrm{C}_{\max }$ y $\mathrm{Fm} /$ block/ $\mathrm{C}_{\max }$. Dicha extensión se debe a nuestra preocupación por acercar y adaptar algunos problemas académicos a la realidad presente en entornos industriales vinculados al sector de automoción.

Nuestra extensión tiene en cuenta la tipología de trabajos o productos en dichos problemas, ante el hecho evidente de que, en muchos sectores industriales altamente estandarizados como lo es el de automoción, es improbable encontrar procesos productivos donde todos los trabajos o todos los productos sean completamente distintos. Por ello, hemos incorporado a los problemas originales el concepto de plan de demanda de tipos de trabajo o tipos de producto $\left(\mathrm{d}_{\mathrm{i}} \forall \mathrm{i} \in \mathrm{I}\right)$, dando lugar a los problemas Fm/prmu/ $\mathrm{C}_{\max } / \mathrm{d}_{\mathrm{i}}$ y Fm/block/ $\mathrm{C}_{\max } / \mathrm{d}_{\mathrm{i}}$, siendo, por supuesto, dicho concepto extrapolable a otras variantes de problemas de secuenciación, cuando se den las circunstancias propicias.

Tras formular los modelos de Programación lineal entera mixta para los dos nuevos problemas, hemos realizado una experiencia computacional, asistida por el solucionador CPLEX, sobre el conjunto de instancias Nissan-9Eng.I. El propósito de dicho experimento ha sido analizar la posibilidad de transformar una línea de modelos mixtos de motores en un taller de flujo regular considerando flujos de producción sin bloqueo (prmu) y con bloqueo (block) entre estaciones de trabajo. Tal experimentación pone de manifiesto las ventajas desde la óptica productiva que ofrece un taller de flujo regular sin bloqueos frente a la línea de motores mixtos original.

Posteriormente, refiriéndonos a los aspectos tecnológicos que afectan a las soluciones, hemos descrito los requisitos para llevar a la práctica una secuencia de fabricación $\pi(\mathrm{T})$ garantizando el ritmo que marca un sistema productivo del tipo taller de flujo regular. Para esto último, proponemos el uso de tecnologías IoT en el marco de la Industria 4.0.

Sobre la Programación Lineal Entera Mixta, concluimos que no existe razón suficiente para descartar su uso frente a problemas de secuenciación en entornos productivos tipo taller de flujo regular, puesto que resulta ser una técnica competitiva para $\mathrm{Fm} / \mathrm{prmu} / \mathrm{C}_{\text {max }} / \mathrm{d}_{\mathrm{i}}$ ante instancias de dimensiones industriales de 270 trabajos de 9 tipos con 21 estaciones de trabajo, obteniendo y confirmando las soluciones óptimas en todos los planes del conjunto Nissan-9Eng.I en menos de 20 segundos por instancia.

Por su parte, tal técnica se comporta con menor eficacia ante el problema Fm/block/ $C_{\max } / d_{i}$ con el mismo conjunto de instancias industriales. No obstante, los resultados se pueden calificar como aceptables, ya que se obtienen soluciones en 3 minutos de CPU que están en promedio al 1,49\% de la mejor cota inferior confirmada.
Por lo anterior, concluimos que MILP debe incorporarse al conjunto de herramientas dedicadas a resolver problemas de secuenciación en entornos productivos realistas. El rol de MILP dentro del conjunto de técnicas de resolución para dichos problemas puede ser como protagonista o como parte de procedimientos metaheurísticos que combinan una fase de construcción de una o varias soluciones iniciales con una o varias fases de mejora local de dichas soluciones.

Finalmente, en líneas futuras de trabajo, proponemos incorporar a los modelos presentados otros conceptos productivos como el factor de actividad de los operarios y algunas propiedades de la secuenciación de modelos mixtos requeridas en contexto Just-in-Time.

\section{Agradecimientos}

Esta investigación ha sido subvencionada por el Ministerio de Ciencia, Innovación y Universidades del Gobierno de España con el proyecto OPTHEUS (ref. PGC2018-095080B-I00), incluyendo Fondos para el desarrollo regional europeo (ERDF: European Regional Development Funds).

\section{Referencias}

Aggoune R (2004) Minimizing the makespan for the flow shop scheduling problem with availability constraints. Eur J Oper Res 153(3):534-543. https://doi.org/10.1016/ S0377-2217(03)00261-3

Bautista J, Cano A (2011) Solving mixed model sequencing problem in assembly lines with serial workstations with work overload minimisation and interruption rules. Eur J Oper Res 210(3):495-513. https://doi.org/10.1016/j. ejor.2010.10.022

Bautista-Valhondo J, Alfaro-Pozo R (2018a) Mixed integer linear programming models for flow shop scheduling with a demand plan of job types. Cen Eur J Oper Res. https://doi.org/10.1007/s10100-018-0553-8

Bautista-Valhondo J, Alfaro-Pozo R (2018b) An expert system to minimize operational costs in mixed-model sequencing problems with activity factor. Expert Syst Appl 104:185-201. https://doi.org/10.1016/j. eswa.2018.03.031

Bautista J, Cano A, Companys R, Ribas I (2012) Solving the Fm|block|Cmax problem using Bounded Dynamic Programming. Eng Appl Artif Intell 25(6):1235-1245. https://doi.org/10.1016/j.engappai.2011.09.001

Bautista J., Alfaro-Pozo R., Batalla-García C. (2018) Minimizing Lost-Work Costs in a Mixed-Model Assembly Line. In: Viles E., Ormazábal M., Lleó A. (eds) Closing the Gap Between Practice and Research in Indus- 
trial Engineering. Lecture Notes in Management and Industrial Engineering. Springer, Cham. https://doi. org/10.1007/978-3-319-58409-6_24

Caraffa V, Ianes S, Bagchi TP, Sriskandarajah C (2001) Minimizing makespan in a blocking flowshop using genetic algorithms. Int J Prod Econ 70(2):101-115. https://doi. org/10.1016/S0925-5273(99)00104-8

Fernandez-Viagas V, Ruiz R, Framinan JM (2017) A new vision of approximate methods for the permutation flowshop to minimise makespan: state-of-the-art and computational evaluation. Eur J Oper Res 257(3):707-721. https://doi.org/10.1016/j.ejor.2016.09.055

Grabowski J, Pempera J (2007) The permutation flow shop problem with blocking. A tabu search approach. Omega 35(3):302-311. https://doi.org/10.1016/j.omega.2005.07.004

Graham RL, Lawler EL, Lenstra JK, Rinnooy Kan AHG (1979) Optimization and approximation in deterministic sequencing and scheduling: a survey. Ann Discret Math 5:287-326. https://doi.org/10.1016/S01675060(08)70356-X

Hall NG, Sriskandarajah C (1996) A survey of machine scheduling problems with blocking and no-wait in process. Oper Res 44(3):510-525. https://doi.org/10.1287/ opre.44.3.510

Han YY, Pan QK, Li JQ et al (2012) An improved artificial bee colony algorithm for the blocking flowshop scheduling problema. Int J Adv Manuf Technol 60:1149-1159. https://doi.org/10.1007/s00170-011-3680-0

Hoogeveen JA, Lenstra JK, Veltman B (1996) Preemptive scheduling in a two-stage multiprocessor flow shop is NP-hard. Eur J Oper Res 89(1):172-175. https://doi. org/10.1016/S0377-2217(96)90070-3

Lin SW, Ying KC (2013) Minimizing makespan in a blocking flowshop using a revised artificial immune system algorithm. Omega 41(2):383-389. https://doi.org/10.1016/j. omega.2012.03.006

Logendran R, Sriskandarajah C (1993) Two-machine group scheduling problem with blocking and anticipatory setups. Eur J Oper Res 69(3):467-481. https://doi. org/10.1016/0377-2217(93)90029-M

Nawaz M, Enscore EE, Ham I (1983) A heuristic algorithm for the m-machine, n-job flow-shop sequencing problema. Omega 11(1):91-95. https://doi.org/10.1016/03050483(83)90088-9
Nouri N, Ladhari T (2018) Evolutionary multiobjective optimization for the multi-machine flow shop scheduling problem under blocking. Ann Oper Res 267:413-430. https://doi.org/10.1007/s10479-017-2465-8

Osman IH, Potts CN (1989) Simulated annealing for permutation flow-shop scheduling. Omega 17(6):551-557. https://doi.org/10.1016/0305-0483(89)90059-5

Ozolins A (2019) Improved bounded dynamic programming algorithm for solving the blocking flow shop problem. Cent Eur J Oper Res 27(1):15-38. https://doi. org/10.1007/s10100-017-0488-5

Pan QK, Wang L (2012) Effective heuristics for the blocking flowshop scheduling problem with makespan minimization. Omega 40(2):218-229. https://doi.org/10.1016/j. omega.2011.06.002

Pinedo ML (2016) Scheduling (theory, algorithms, and systems). Springer International Publishing, Berlin. https:// doi.org/10.1007/978-3-319-26580-3

Reeves CR (1995) A genetic algorithm for flowshop sequencing. Comput Oper Res 22(1):5-13. https://doi. org/10.1016/0305-0548(93)E0014-K

Ribas I, Companys R, Tort-Martorell X (2011) An iterated greedy algorithm for the flowshop scheduling problem with blocking. Omega 39(3):293-301. https://doi. org/10.1016/j.omega.2010.07.007

Ronconi DP (2004) A note on constructive heuristics for the flowshop problem with blocking. Int J Prod Econ 87(1):39-48. https://doi.org/10.1016/S09255273(03)00065-3

Ronconi DP (2005) A branch-and-bound algorithm to minimize the makespan in a flowshop with blocking. Ann Oper Res 138(1):53-65. https://doi.org/10.1007/ s10479-005-2444-3

Taillard E(1990) Some efficient heuristic methods for the flow shop sequencing problema. Eur J Oper Res 47(1):65-74. https://doi.org/10.1016/0377-2217(90)90090-X

Taillard E (1993) Benchmarks for basic scheduling problems. Eur J Oper Res 64(2):278-285. https://doi. org/10.1016/0377-2217(93)90182-M

Tasgetiren MF, Kizilay D, Pan QK, Suganthanc PN (2017) Iterated greedy algorithms for the blocking flowshop scheduling problem with makespan criterion. Comput Oper Res 77:111-126. https://doi.org/10.1016/j. cor.2016.07.002 
Ying KC, Liao CJ (2004) An ant colony system for permutation flow-shop sequencing. Comput Oper Res 31(5): 791801. https://doi.org/10.1016/S0305-0548(03)00038-8
Yu W, Hoogeveen H, Lenstra JK (2004) Minimizing makespan in a two-machine flow shop with delays and unit-time operations is NP-hard. J Sched 7(5):333-348. https://doi.org/10.1023/B:JOSH.0000036858.59787.c2 